\title{
Phosphorus forms in forest soil colloids as revealed by liquid-state ${ }^{31}$ P-NMR
}

\section{Supplementary Material}

Author list

Anna Missong ${ }^{1}$, Roland Bol ${ }^{1}$, Sabine Willbold ${ }^{2}$, Jan Siemens ${ }^{3}$ and Erwin Klumpp ${ }^{1}$

Affiliations

${ }^{1}$ Institute of Bio- and Geosciences, Agrosphere (IBG-3), Research Centre Jülich, Wilhelm Johnen Str., 52425 Jülich, Germany

${ }^{2}$ Central Institute for Engineering, Electronics and Analytics, Analytics (ZEA-3), Research Centre Jülich, Wilhelm Johnen Str., 52425 Jülich, Germany

${ }^{3}$ Institute of Soil Science and Soil Conservation, Justus-Liebig University Giessen, Heinrich-Buff-Ring 26-32, 35392 Gießen, Germany 


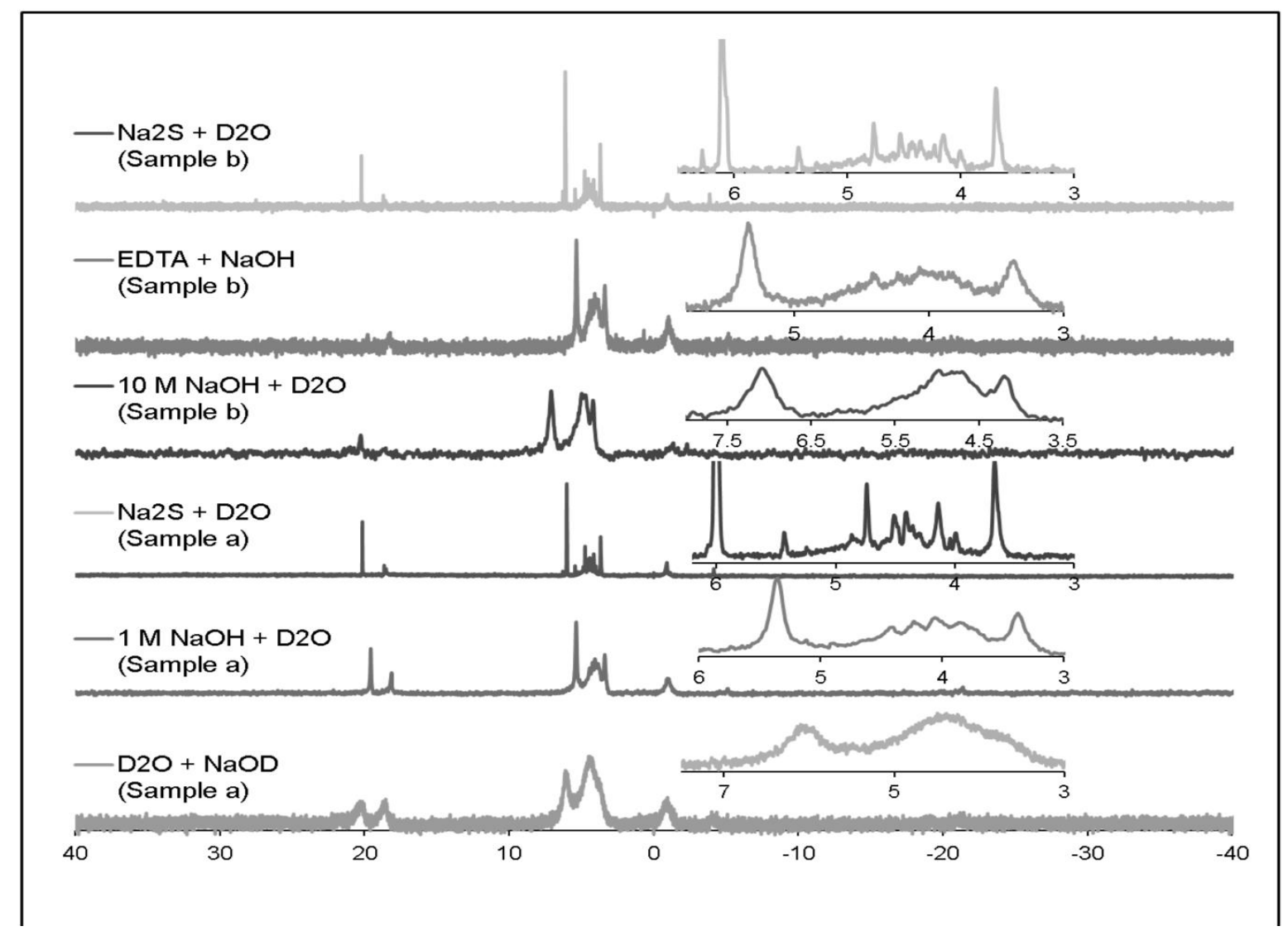

Figure S1: Comparison of re-dissolving tests for the acidic forest bulk soil samples. Two different samples were used (sample a: of an Oh-horizon and sample b: of an Ah-horizon).

Sample a: The spectra below showed the sample re-dissolved in $1.5 \mathrm{~mL} \mathrm{D}_{2} \mathrm{O}$ and $100 \mu \mathrm{L} \mathrm{NaOD}$. The second sample was re-dissolved in $1 \mathrm{~mL}$ of $1 \mathrm{M} \mathrm{NaOH}+100 \mu \mathrm{L}_{2} \mathrm{O}$. The third spectra shows the result of the redissolving as performed for the samples in the manuscript, with $1.5 \mathrm{~mL}$ of $5 \mathrm{M} \mathrm{Na}_{2} \mathrm{~S}$ (in $\mathrm{D}_{2} \mathrm{O}$ ) and $10 \mu \mathrm{L} \mathrm{NaOD}$. Sample b: The fourth samples was dissolved in $10 \mathrm{M} \mathrm{NaOH}+200 \mu \mathrm{L} \mathrm{D}_{2} \mathrm{O}$. The fifth spectra is of a sample dissolved in $1 \mathrm{M} \mathrm{NaOH}+0.1 \mathrm{M}$ EDTA $+200 \mu \mathrm{L} \mathrm{D}_{2} \mathrm{O}$. To compare the signal quality the y-axes intensity was adjusted. The upper spectra is from sample b dissolved in $1.5 \mathrm{~mL}$ of $5 \mathrm{M} \mathrm{Na}_{2} \mathrm{~S}$ (in $\mathrm{D}_{2} \mathrm{O}$ ) and $10 \mu \mathrm{LaOD}$.

Table S1: Relative proportion P compound classes for the three spectra shown in Figure 1. The proportions were calculated by the integration of the signal shapes. The sample extracted with D2O and NaOD does not show quantitative results because the signal to noise ratio was too low.

Phosphonate Phosphonate

1

2

Ortho-P Monoester-P Diester-P Pyro-P Poly- $\mathrm{P}$

\begin{tabular}{|c|c|c|c|c|c|c|c|}
\hline sample & \multicolumn{7}{|c|}{ 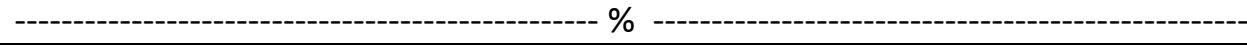 } \\
\hline b) $5 \mathrm{M} \mathrm{Na} 2 \mathrm{~S}$ in $\mathrm{D} 2 \mathrm{O} \& \mathrm{NaOD}$ & 4 & 3 & 23 & 60 & 9 & 1 & 0 \\
\hline b) $10 \mathrm{M} \mathrm{NaOH} \& \mathrm{D} 2 \mathrm{O}$ & 1 & 3 & 21 & 59 & 14 & 2 & 0 \\
\hline b) $1 \mathrm{M} \mathrm{NaOH}+0.1 \mathrm{M} \mathrm{EDTA}+\mathrm{D} 2 \mathrm{O}$ & 3 & 0 & 29 & 63 & 5 & 0 & 0 \\
\hline a) $5 \mathrm{M} \mathrm{Na} 2 \mathrm{~S}$ in $\mathrm{D} 2 \mathrm{O} \& \mathrm{NaOD}$ & 6 & 5 & 20 & 56 & 11 & 1 & 1 \\
\hline a) $1 \mathrm{M} \mathrm{NaOH} \& \mathrm{D} 2 \mathrm{O}$ & 8 & 6 & 23 & 51 & 10 & 1 & 1 \\
\hline a) $\mathrm{D} 2 \mathrm{O} \& \mathrm{NaOD}$ & 8 & 6 & 21 & 53 & 9 & 1 & 2 \\
\hline
\end{tabular}


Table S2: Control of the P, Fe and Mn concentrations of the NMR samples redissolved in three different ways. Firstly, in the same way the samples (of this work) were treated: $1 \mathrm{~mL}$ of $5 \mathrm{M} \mathrm{Na}_{2} \mathrm{~S}$ (in $\mathrm{D}_{2} \mathrm{O}$ ) and $10 \mu \mathrm{L} \mathrm{NaOD;secondly,} \mathrm{the} \mathrm{same}$ samples were redissolved in $10 \mathrm{M} \mathrm{NaOH}+200 \mu \mathrm{L} \mathrm{D}_{2} \mathrm{O}$, and thirdly exemplary the bulk soil samples were redissolved in $1 \mathrm{M} \mathrm{NaOH}+0.1 \mathrm{M}$ EDTA $+200 \mu \mathrm{L} \mathrm{D}_{2} \mathrm{O}$. The concentrations show that the $\mathrm{Na}_{2} \mathrm{~S}$ mostly increased the $\mathrm{P}$ concentrations in the sampled but reduce the Fe and $\mathrm{Mn}$ concentrations. STD means standard deviation.

\begin{tabular}{|c|c|c|c|c|c|c|c|c|}
\hline & & $\begin{array}{c}\mathrm{P} \\
\mathrm{mg} / \mathrm{g} \\
\text { sample }\end{array}$ & $\begin{array}{l}\text { STD } \\
\mathrm{mg} / \mathrm{g} \\
\text { sample }\end{array}$ & $\begin{array}{c}\mathrm{Fe} \\
\mathrm{mg} / \mathrm{g} \\
\text { sample }\end{array}$ & $\begin{array}{c}\text { STD } \\
\mathrm{mg} / \mathrm{g} \\
\text { sample }\end{array}$ & $\begin{array}{c}\mathrm{Mn} \\
\mathrm{mg} / \mathrm{g} \\
\text { sample }\end{array}$ & $\begin{array}{c}\text { STD } \\
\mathrm{mg} / \mathrm{g} \\
\text { sample }\end{array}$ & $\mathrm{P} /(\mathrm{Fe}+\mathrm{Mn})$ \\
\hline \multirow{12}{*}{ 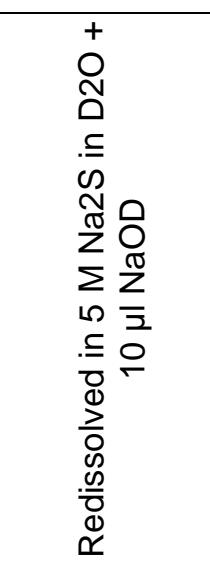 } & \multirow{5}{*}{ 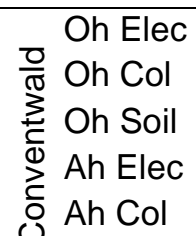 } & 0.06 & 0.003 & 0.01 & 0.002 & 0.005 & 0.000 & 5.23 \\
\hline & & 0.25 & 0.02 & 0.03 & 0.003 & 0.001 & 0.000 & 9.43 \\
\hline & & 0.93 & 0.42 & 0.14 & 0.085 & 0.001 & 0.000 & 6.84 \\
\hline & & 0.07 & 0.002 & 0.01 & 0.000 & 0.017 & 0.001 & 3.10 \\
\hline & & 0.09 & 0.002 & 0.01 & 0.001 & 0.004 & 0.000 & 6.51 \\
\hline & \multirow{2}{*}{$\begin{array}{l}\text { Ah Soil } \\
\text { Oh Elec }\end{array}$} & 0.38 & 0.02 & 0.05 & 0.003 & 0.001 & 0.000 & 7.38 \\
\hline & & 0.03 & 0.00 & 0.00 & 0.001 & 0.000 & 0.000 & 6.03 \\
\hline & \multirow{3}{*}{$\begin{array}{l}\frac{c}{0} \text { Oh Col } \\
\widetilde{\pi} \text { Oh Soil } \\
\frac{0}{0} \text { Oh So } \\
\frac{\Phi}{\omega} \text { Ah Elec }\end{array}$} & 0.33 & 0.01 & 0.04 & 0.001 & 0.001 & 0.000 & 8.63 \\
\hline & & 0.57 & 0.07 & 1.09 & 0.538 & 0.034 & 0.002 & 0.51 \\
\hline & & 0.03 & 0.00 & 0.01 & 0.001 & 0.000 & 0.000 & 4.77 \\
\hline & \multirow{2}{*}{$\begin{array}{l}\text { Ah Col } \\
\text { Ah Soil }\end{array}$} & 0.25 & 0.01 & 0.03 & 0.001 & 0.000 & 0.000 & 8.09 \\
\hline & & 0.33 & 0.02 & 0.61 & 0.179 & 0.211 & 0.002 & 0.40 \\
\hline \multirow{12}{*}{ 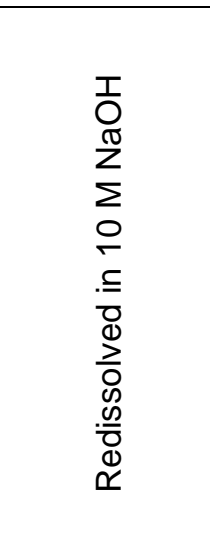 } & \multirow{5}{*}{ 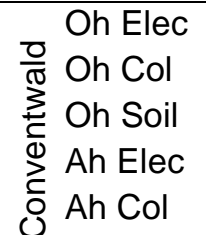 } & 0.03 & 0.001 & 0.21 & 0.004 & 0.009 & 0.000 & 0.16 \\
\hline & & 0.17 & 0.01 & 1.38 & 0.102 & 0.018 & 0.002 & 0.12 \\
\hline & & 0.73 & 0.02 & 1.41 & 0.398 & 0.052 & 0.002 & 0.50 \\
\hline & & 0.05 & 0.002 & 0.20 & 0.008 & 0.025 & 0.002 & 0.23 \\
\hline & & 0.08 & 0.01 & 0.24 & 0.015 & 0.010 & 0.001 & 0.32 \\
\hline & Ah Soil & 0.28 & 0.01 & 2.00 & 0.110 & 0.024 & 0.002 & 0.14 \\
\hline & Oh Elec & 0.03 & 0.01 & 0.13 & 0.004 & 0.002 & 0.000 & 0.22 \\
\hline & \multirow{2}{*}{ 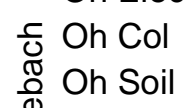 } & 0.25 & 0.02 & 0.55 & 0.042 & 0.007 & 0.000 & 0.44 \\
\hline & & 0.66 & 0.52 & 2.95 & 0.052 & 0.021 & 0.010 & 0.22 \\
\hline & \multirow{2}{*}{ 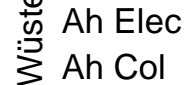 } & 0.01 & 0.001 & 0.04 & 0.001 & 0.001 & 0.000 & 0.33 \\
\hline & & 0.16 & 0.005 & 0.16 & 0.007 & 0.003 & 0.000 & 0.97 \\
\hline & Ah Soil & 0.44 & 0.02 & 0.70 & 0.028 & 0.210 & 0.004 & $\underline{0.48}$ \\
\hline \multirow{4}{*}{ 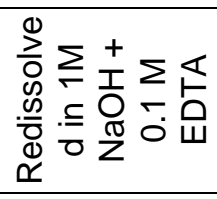 } & \multirow{4}{*}{$\begin{array}{l}3 \text { Oh Soil } \\
\text { U Ah Soil } \\
\sum_{3} \text { Oh Soil } \\
\text { Ah soil }\end{array}$} & 0.05 & 0.002 & 0.06 & 0.001 & 0.005 & 0.000 & $\overline{0.71}$ \\
\hline & & 0.46 & 0.02 & 7.94 & 0.196 & 0.047 & 0.001 & 0.06 \\
\hline & & 1.02 & 0.55 & 6.54 & 0.157 & 0.039 & 0.016 & 0.16 \\
\hline & & 0.36 & 0.03 & 1.27 & 0.318 & 0.121 & 0.013 & 0.26 \\
\hline
\end{tabular}


2) Method development: ${ }^{31} \mathrm{P}-\mathrm{NMR}$ Measurement parameters

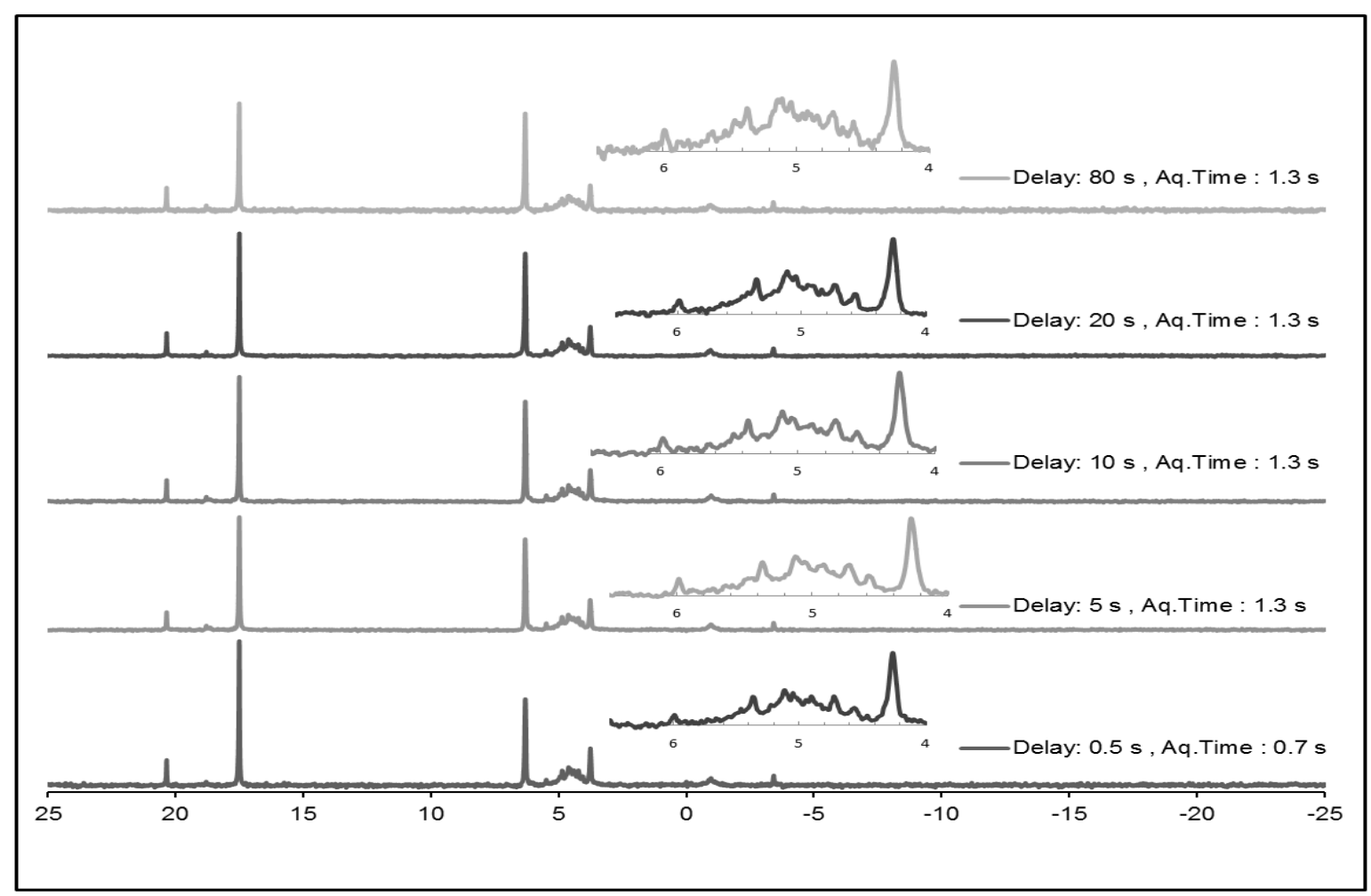

Figure S2: Spectra measured at different 31P-NMR measurement parameters. The under most spectra demonstrates the experimental parameters used in the paper. An Oh-horizon sample was used for the measurement.

Table S3: Relative proportion P compound classes for the five spectra shown in Figure 2. The proportions were calculated by the integration of the signal shapes.

\begin{tabular}{|c|c|c|c|c|c|c|c|}
\hline & & Phosphonates & MDPA & Ortho-P & Monoester & Diester & Pyro- \& Poly-P \\
\hline \multirow{5}{*}{$\begin{array}{c}\text { Delay Time, } \\
\text { Aq. Time }\end{array}$} & $80 \mathrm{~s}, 1.3 \mathrm{~s}$ & 5 & 20 & 25 & 43 & 2 & 5 \\
\hline & $20 \mathrm{~s}, 1.3 \mathrm{~s}$ & 4 & 21 & 25 & 43 & 2 & 5 \\
\hline & $10 \mathrm{~s}, 1.3 \mathrm{~s}$ & 5 & 21 & 23 & 44 & 1 & 6 \\
\hline & $5 \mathrm{~s}, 1.3 \mathrm{~s}$ & 5 & 21 & 23 & 45 & 1 & 5 \\
\hline & $0.5 \mathrm{~s}, 0.7 \mathrm{~s}$ & 4 & 22 & 20 & 46 & 2 & 6 \\
\hline
\end{tabular}

